\title{
Synthesis, structure, and chemistry of selenium-containing four-membered rings *
}

\author{
Eric Block ${ }^{\ddagger}$, Evgeny V. Dikarev, Jin Jin, Bo Li, Marina A. Petrukhina, \\ and Shao-Zhong Zhang
}

Department of Chemistry, University at Albany, SUNY, Albany, NY 12222, USA

\begin{abstract}
Recent chemistry of four-membered ring selenium compounds is reviewed followed by a summary of work in our laboratories in this area. 2-Thia-6-selenaspiro[3.3]heptane has been synthesized for the first time. X-ray structures for the latter compound as well as for 2,6-dithiaspiro[3.3] heptane and 2,6-diselenaspiro[3.3]heptane have been determined. These compounds are useful as "rigid-rod" ligands for coordination to transition-metal complexes.
\end{abstract}

Keywords: Selenium; four-membered rings; X-ray structures; metal complexes; selenetane.

\section{INTRODUCTION}

Thietanes and related sulfur-containing four-membered rings are well-studied heterocycles of interest as natural substances, products of commerce, novel ligands in transition-metal complexes, substrates for organic synthesis, and photochemical and pyrolytic precursors for heteroatom species $R_{2} M=X$ [1]. In contrast, selenetanes and related selenium-containing four-membered rings have been relatively little studied [1d,2]. We have initiated a research program to synthesize and study new chalcogen-containing heterocycles, including those with four-membered rings, and their metal complexes. We describe here recent work in our laboratory on one novel class of selenium-containing four-membered rings, namely, the 2,6-dichalcogenospiro[3.3] heptanes. This work is presented in the context of work in other laboratories on selenium-containing four-membered rings and their metal complexes, which will first be briefly reviewed.

The structure of selenetane has been determined by microwave spectroscopy [3a], and the synthesis, oxidation, anionic polymerization, and other aspects of the chemistry of 3,3-dimethylselenetane and other 3 -substituted selenetanes have been reported [3b-e]. Adams has developed a useful approach to the synthesis of mesocyclic polyselenacycloalkanes such as 1,5,9-triselenacyclododecane using rhenium carbonyl complexes of 3,3-dimethylselenetane as catalysts [3f,g], while Fischer has prepared tungsten complexes of selenetanes by thermal cycloaddition of vinyl ethers to transition-metal-coordinated selenoaldehydes [3h]. The Taxol ${ }^{\circledR}$ oxetane ring has been replaced by a selenetane ring [3i], several studies of 1,3-diselenetanes have appeared [3j,k], and syntheses of a 1,3-disila-2,4-diselenetane $\left(\mathrm{R}_{2} \mathrm{Si}=\mathrm{Se}\right.$ dimer) [31], a 1-selena-2-siletane [3m], a selenathietane [3n], and 1,3-oxaselenetanes [3o,p] are reported. Other recent papers report the isolation of a $2 \mathrm{H}$-benzoselenete [3q], metal complexes of a $2 \mathrm{H}$-selenete [3r], synthesis and X-ray structure of a selenet-2(2H)-imine [3s], synthesis of novel struc-

\footnotetext{
*Paper based on a presentation at the $7^{\text {th }}$ IUPAC International Conference on Heteroatom Chemistry (ICHAC-7), Shanghai, China, 21-25 August 2004. Other presentations are published in this issue, pp. 1985-2132.

¥Corresponding author
} 
tures containing selenetanes fused to furanoside rings [ $3 \mathrm{t}, \mathrm{u}]$, and general new methods for the synthesis of selenetanes $[3 \mathrm{v}]$.

We have previously synthesized and explored the chemistry of 4- and 6-membered rings containing two sulfur or selenium atoms, such as 1,3-dithietane, 1, and 1,2-dithiin, 1,2-diselenin, and 2-selenathiin, 2 [4-7] as well as the structure and coordination chemistry of small acyclic organoselenium compounds including dimethyl diselenide [8], dimethyl selenoxide [9], and dimethyl selenone [10]. We sought to extend these studies to other unknown or little studied heterocycles containing sulfur and/or selenium, particularly those containing the selenetane ring, whose coordination chemistry has been scarcely studied. Here, we report the synthesis and characterization of the previously unknown heterocycle, 2-thia-6-selenaspiro[3.3]heptane, $\mathbf{3}$, as well as the known, but incompletely characterized 2,6-diselenaspiro[3.3]heptane, 5 [3b] and the better studied 2,6-dithiaspiro[3.3]heptane, 4 [11]. Compounds 3-5 are useful as "rigid-rod" ligands with a sigma-bonded framework.

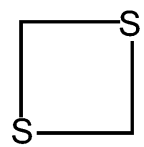

1<smiles></smiles>

2, $\mathrm{E}=\mathrm{S}$ and/or Se

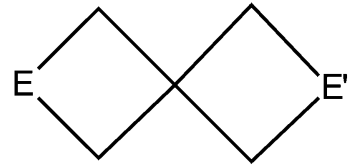

3, $E=S, E^{\prime}=S e$

4, $E=E^{\prime}=S$

5, $E=E^{\prime}=S e$

\section{RESULTS AND DISCUSSION}

Heterocycle $\mathbf{3}$ was synthesized from 1,3-dibromo-2,2-bis(bromomethyl)propane, $\mathbf{6}$, (Scheme 1) by replacement of one of the bromines with thioacetate, treatment with base to give 3,3-bis(bromomethyl)thietane, 7, and conversion of 7 to 3 by $\mathrm{Li}_{2} \mathrm{Se}$ (from $\mathrm{Se}$ and $\mathrm{LiEt}_{3} \mathrm{BH}$ ) in THF $[12,13]$. Heterocycle $\mathbf{4}$ was prepared as described previously [11]. Heterocycle $\mathbf{5}$ was prepared in $70 \%$ yield by reaction of $\mathrm{Li}_{2} \mathrm{Se}$ in refluxing THF with $\mathbf{6}$. Selected data for $\mathbf{3}, \mathbf{4}$, and $\mathbf{5}$ (all colorless solids) are given: 3, m.p. $45{ }^{\circ} \mathrm{C},{ }^{1} \mathrm{H}$ NMR $\delta 3.16$ (s, 4H), 3.18 (s, 4H), ${ }^{13} \mathrm{C}$ NMR $\delta 27.3,41.1,53.3 ; 4$, m.p. $31{ }^{\circ} \mathrm{C}$, ${ }^{1} \mathrm{H}$ NMR $\delta 3.26$ (s), ${ }^{13} \mathrm{C}$ NMR $\delta 39.8,52.2 ; 5$, m.p. $68{ }^{\circ} \mathrm{C},{ }^{1} \mathrm{H}$ NMR $\delta 3.14$ (s), ${ }^{13} \mathrm{C}$ NMR $\delta 28.7,54.4$. $\mathrm{X}$-ray structures for $\mathbf{3}$ and $\mathbf{4}$ are shown in Fig. 1; data appear in Table 1 [13]. X-ray structures for metalfree selenetanes have not been previously reported. The coordination chemistry of 3-5 as rigid-rod ligands toward complexes of cobalt, copper, nickel, and rhodium, and full X-ray structure of $\mathbf{5}$ have been

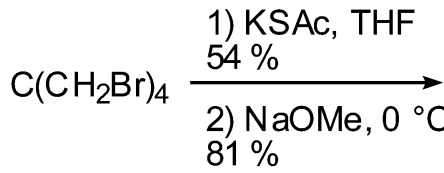

6

Scheme 1
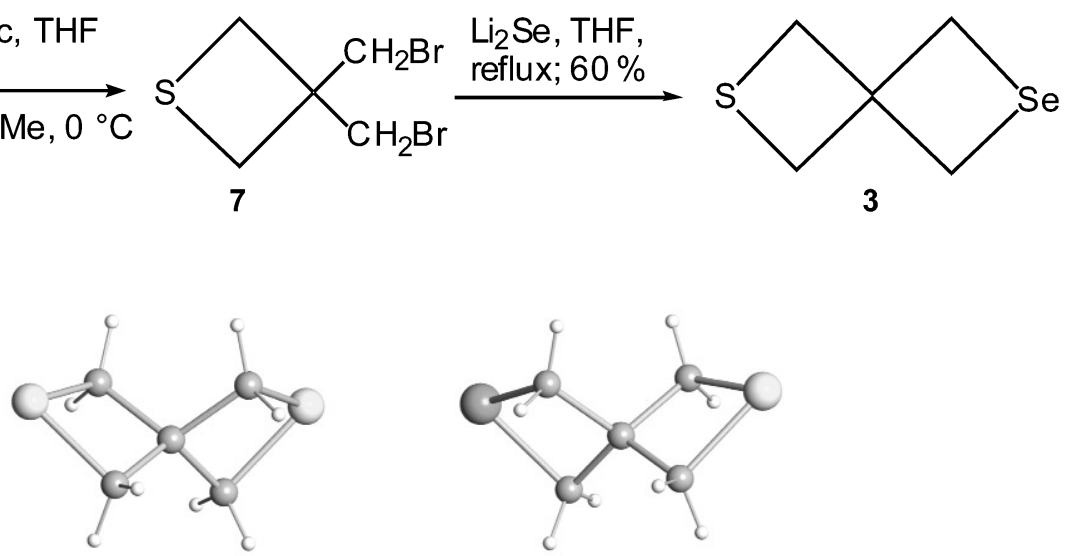

Fig. 1 X-ray crystal structures of $\mathbf{4}$ (left) and $\mathbf{3}$ (right; the Se atom is on the left in $\mathbf{3}$ ). 
reported [14]. A study of magnetic properties shows that complexes of 5 do not facilitate magnetic interactions between paramagnetic metal centers [13].

Table 1 Selected distances $(\AA)$ and angles $\left(^{\circ}\right)$ in 2,6-diheterospiro[3.3]heptanes 3-5.

\begin{tabular}{llll}
\hline & \multicolumn{1}{c}{$\mathbf{3}^{*}$} & \multicolumn{1}{c}{$\mathbf{4}$} & \multicolumn{1}{c}{$\mathbf{5}$} \\
\hline $\mathrm{E}-\mathrm{C}_{\mathrm{av}}$ & $1.908(6)$ & $1.835(1)$ & $1.978(3)$ \\
$\mathrm{C}-\mathrm{C}_{\mathrm{av}}$ & $1.535(2)$ & $1.536(2)$ & $1.537(4)$ \\
$\mathrm{E} \bullet \bullet \mathrm{E}$ & $4.792(6)$ & $4.690(1)$ & $4.906(1)$ \\
$\mathrm{C}-\mathrm{E}-\mathrm{C}_{\mathrm{av}}$ & $74.1(1)$ & $76.65(6)$ & $72.0(1)$ \\
$\mathrm{C}-\mathrm{C}-\mathrm{C}$ & $97.0(1)-120.0(1)$ & $96.1(1)-121.1(1)$ & $98.1(2)-119.1(2)$ \\
\hline
\end{tabular}

*Average for the two mixed positions

\section{ACKNOWLEDGMENTS}

We acknowledge the support of the National Science Foundation (CHE-9906566 and CHE-0450505, E.B.; CHE-01300985, E. D.) and the Donors of the American Chemical Society Petroleum Research Fund (38450-AC1, E.B; 39039-G3, M. P.).

\section{REFERENCES}

1. (a) E. Block. Compr. Heterocycl. Chem. I 5, 403-447 (1984); (b) E. Block and M. De Wang. Compr. Heterocycl. Chem. II 1B, 774-802 (1996); (c) E. Block and M. De Wang. Compr. Heterocycl. Chem. II 1B, 803-821 (1996); (d) E. Block and R. J. Linderman. Rodd's Chem. Carbon Compd., $2^{\text {nd }}$ ed., 4 (Pt. A), 113-145 (1997).

2. M. R. Detty. Compr. Heterocycl. Chem. II 1B, 823-831 (1996).

3. (a) M. G. Petit, J. S. Gibson, D. O. Harris. J. Chem. Phys. 53, 3408-3413 (1970); (b) H. J. Backer and H. J. Winter. Rec. Trav. Chim. 56, $492-509$ (1937); (c) B. Lindgren. Chem. Scr. 16, 24-27 (1980); (d) G. Polson and D. C. Dittmer. J. Org. Chem. 53, 791-794 (1988); (e) E. J. Goethals, E. Schacht, D. Tack. J. Polym. Sci., Part A-1, 10, 533-539 (1972); (f) R. D. Adams and K. T. McBride. Chem. Commun. 525-526 (1997); (g) R. D. Adams, K. T. McBride, R. D. Rogers. Organometallics 16, 3895-3901 (1997); (h) H. Fischer, K. Treier, C. Troll. Chem. Ber. 128, 1149-1156 (1995); (i) A. A. L. Gunatilaka, F. D. Ramdayal, M. H. Sarragiotto, D. G. I. Kingston, D. L. Sackett, E. Hamel. J. Org. Chem. 64, 2696-2703 (1999); (j) G. Rabe, K. Keller, H. W. Roesky, R. J. Lagow, F. Pauer, D. Stalke. Z. Naturforsch. B46, 157-160 (1991); (k) T. G. Back, B. P. Dyck, M. Parvez. J. Org. Chem. 60, 703-710 (1995); (l) D. P. Thompson and P. Boudjouk. J. Chem. Soc., Chem. Commun. 1466-1467 (1987); (m) P. Boudjouk, E. Black, R. Kumarathasan, U. Samaraweera, S. Castellino, J. P. Oliver, J. W. Kampf. Organometallics 13, 3715-3727 (1994); (n) Yu. V. Migalina, V. G. Lend'el, A. S. Koz'min, N. S. Zefirov. Khim. Geterotsikl. Soedin. 5, 708 (Chem. Abstr. 89, 109182, 1978) (1978); (o) M. X. Ding, A. Ishii, J. Nakayawa, M. Hoshino. Bull. Chem. Soc. Jpn. 66, 1714-1721 (1993); (p) A. Ishii, M. X. Ding, J. Nakayama, M. Hoshino. J. Chem. Soc., Chem. Commun. 7-8 (1992); (q) K. Okuma, A. Okada, Y. Koga, Y. Yokomori. J. Am. Chem. Soc. 123, 7166-7167 (2001); (r) H. Fischer, K. Treier, C. Troll, R. Stumpf. Chem. Commun. 2461-2463 (1995); (s) P. K. Atanassov, A. Linden, H. Heimgartner. Heterocycles 62, 521-533 (2004); (t) O. Schulze, J. Voss, G. Adiwidjaja, F. Olbrich. Carbohydr. Res. 339, 1787-1802 (2004); (u) G. Adiwidjaja, O. Schulze, J. Voss, J. Wirsching. Carbohydr. Res. 325, 107-119 (2000); (v) K. Takemura, K. Sakano, A. Takahashi, T. Sakamaki, O. Mitsunobu. Heterocycles 47, 633-637 (1998). 
4. E. Block, E. R. Corey, R. E. Penn, T. L. Renken, P. F. Sherwin, H. Bock, T. Hirabayshi, S. Mohmand, B. Solouki. J. Am. Chem. Soc. 104, 3119-3130 (1982).

5. E. Block, M. Birringer, R. DeOrazio, J. Fabian, R. S. Glass, C. Guo, C. He, E. Lorance, Q. Qian, T. B. Schroeder, Z. Shan, M. Thiruvazhi, G. S. Wilson, X. Zhang. J. Am. Chem. Soc. 122, 5052-5064 (2000).

6. R. S. Glass, N. E. Gruhn, D. L. Lichtenberger, E. Lorance, J. R. Pollard, M. Birringer, E. Block, R. DeOrazio, C. He, Z. Shan, X. Zhang. J. Am. Chem. Soc. 122, 5065-5074 (2000).

7. E. D. Lorance, R. S. Glass, E. Block, X. Li. J. Org. Chem. 68, 8110-8114 (2003).

8. P. Groner, C. W. Gillies, J. Z. Gillies, Y. Zhang, E. Block. J. Mol. Spectrosc. 226, 169-181 (2004).

9. (a) E. V. Dikarev, M. A. Petrukhina, X. Li, E. Block. Inorg. Chem. 42, 1966-1972 (2003); (b) A. S. Filatov, E. Block, M. A. Petrukhina. Acta Cryst. C61, o596-o598 (2005).

10. E. V. Dikarev, R. Y. Becker, E. Block, Z. Shan, R. C. Haltiwanger, M. A. Petrukhina. Inorg. Chem. 42, 7098-7105 (2003).

11. (a) H. Fujihah, K. Imaoka, N. Furukawa, S. Oae. J. Chem. Soc., Perkin Trans. 1 333-336 (1986); (b) E. W. Abel, K. G. Orrell, M. C. Poole, V. Sik. Polyhedron 18, 1345-1353 (1999); (c) C. A. Stein and H. Taube. J. Am. Chem. Soc. 103, 693-695 (1981); (d) C. A. Stein, N. A. Lewis, G. Seitz. J. Am. Chem. Soc. 104, 2596-2599 (1982); (e) N. A. Lewis and D. V. Taveras. Adv. Chem. Ser. 226, 197-210 (1990).

12. J. A. Gladysz, J. L. Hornby, J. E. Garbe. J. Org. Chem. 43, 1204-1208 (1978).

13. M. A. Petrukhina, C. Henck, B. Li, E. Block, J. Jin, S.-Z. Zhang, R. Clerac. Inorg. Chem. 44, 77-84 (2005).

14. E. V. Dikarev, R. V. Shpanchenko, K. W. Andreini, E. Block, J. Jin, M. A. Petrukhina. Inorg. Chem. 43, 5558-5563 (2004). 